\title{
A Linear Time Algorithm for Cubic Subgraph of Halin Graphs
}

\author{
Dingjun Lou and Junfu Liu \\ Department of Computer Science \\ Sun Yat-sen University \\ Guangzhou 510275, P. R. China \\ Email: issldj@mail.sysu.edu.cn
}

\begin{abstract}
In this paper, we design a linear time algorithm to determine whether a Halin graph $\mathbf{H}$ has a cubic subgraph $\mathrm{H}^{*}$. If $\mathrm{H}$ has, then the algorithm finds a cubic subgraph $\mathrm{H}^{*}$ in $\mathrm{H}$; otherwise the algorithm answers "No".
\end{abstract} \author{
graph \\ Keywords-Linear time algorithm; cubic subgraph; Halin
}

\section{INTRODUCTION}

A Halin graph $\mathrm{H}$ is defined as follows: First, we embed a tree $\mathrm{T}$ in the plane such that each inner vertex of $\mathrm{T}$ has degree at least 3 ; then we draw a cycle $\mathrm{C}$ through all leaves of $\mathrm{T}$ to form a planar graph. Then $\mathrm{H}=\mathrm{T} \cup \mathrm{C}$ is called a Halin graph, where $\mathrm{T}$ is called the characteristic tree of $\mathrm{H}$ and $\mathrm{C}$ is called the accompanying cycle of $\mathrm{H}$. The simplest Halin graphs are wheels, where $\mathrm{T}$ has only one inner vertex and the other vertices are leaves of T. Suppose a Halin graph $\mathrm{H}$ is not a wheel. If $\mathrm{w}$ is an inner vertex of $\mathrm{T}$ such that all neighbours $v_{1}, v_{2} \ldots, v_{k}$ of $\mathrm{w}$ except one neighbour are leaves of $\mathrm{T}$, then the induced subgraph $\mathrm{H}\left[\{\mathrm{w}\} \cup\left\{v_{1}, v_{2}, \ldots, v_{k}\right\}\right]$ is called a fan of $\mathrm{H}$ and $\mathrm{w}$ is called the center of the fan, where the induced subgraph G[S] of a graph $G$ on a subset $S$ of vertices in $G$ is a subgraph of $G$ consisting of the vertices in $\mathrm{S}$ and the edges of $\mathrm{G}$ with both ends in $\mathrm{S}$.

Halin graphs were introduced by German mathematician Halin [6] as minimally 3-connected planar graphs. It can be used as a model of a network with minimum cost and fault tolerance.

A graph $G$ is Hamiltonian if $G$ has a cycle through all vertices of $G$. A graph $G$ is 1-Hamiltonian, if $G$ is Hamiltonian and deleting each vertex from $G$, the graph is still Hamiltonian. A graph $\mathrm{G}$ is Hamiltonian connected if, for each pair of vertices $u$ and $v$, there is a Hamiltonian path $P$ from $u$ to $v$ in $G$, where $P$ goes through all vertices of $G$. A graph $\mathrm{G}$ is pancyclic, if $\mathrm{G}$ has a cycle $\mathrm{C}$ of length $\mathrm{L}$ for each integer $\mathrm{L}$ such that $3 \leq \mathrm{L} \leq|\mathrm{V}(\mathrm{G})|$.

Bondy [2] proves that every Halin graph $\mathrm{H}$ is 1Hamiltonian. Then Bondy and Lovász [3] prove that, for each integer $\mathrm{L}$ such that $3 \leq \mathrm{L} \leq|\mathrm{V}(\mathrm{H})|$ except possibly for an even integer, a Halin graph $\mathrm{H}$ has a cycle of length L. Lou [8] proves that every Halin graph is Hamiltonian connected.

Let $G$ be a weighted graph with each edge having a positive weight. The weight of a subgraph $\mathrm{K}$ of $\mathrm{G}$ is the sum of weights of all edges of K. The Traveling Salesman
Problem is to find a Hamiltonian cycle $\mathrm{C}$ with minimum weight among all Hamiltonian cycles in $\mathrm{G}$.

The TSP problem for a general graph is an NP-hard problem. However, Cornuejols, Naddef and Pulleyblank [4] give a linear time algorithm to solve TSP for a weighted Halin graph. Li, Lou and $\mathrm{Lu}$ [7] design a linear time algorithm to find a Hamiltonian path with minimum weight between each pair of vertices in a weighted Halin graph.

The Bottleneck TSP of a weighted graph $\mathrm{G}$ is to find a Hamiltonian cycle $\mathrm{C}$ with the weight of each edge of $\mathrm{C}$ less than or equal to a given number B. The Bottleneck TSP is also an NP-Complete problem.

Phillips, Punnen and Kabadi [11] design a linear time algorithm to solve the BTSP for a weighted Halin graph. Lou and Dou [10] design a linear time algorithm to find a Hamiltonian cycle satisfying the bottleneck restriction and having minimum weight in a weighted Halin graph.

Lou and Zhu [9] also give a linear time algorithm to solve another NPC problem, the Max-leaves Spanning Tree Problem, for Halin graphs.

The problem to determine whether a general graph $\mathrm{G}$ has a cubic subgraph $\mathrm{G}^{*}$ such that for every vertex $\mathrm{w}$ of $\mathrm{G}^{*}$, $d_{G^{*}}(w)=3$ is an NPC problem (see [5]). However, for a Halin graph $\mathrm{H}$, the problem to determine whether $\mathrm{H}$ has a cubic subgraph $\mathrm{H}^{*}$ can be solved in linear time. In this paper, we design a linear time algorithm to determine whether a Halin graph $\mathrm{H}$ has a cubic subgraph $\mathrm{H}^{*}$. If $\mathrm{H}$ has, then the algorithm finds a cubic subgraph $\mathrm{H}^{*}$; otherwise the algorithm answers "No". We also prove the correctness of the algorithm and analyze the time complexity of the algorithm. The algorithm is optimal.

In [4], it is mentioned that given a Halin graph $\mathrm{H}$, we can find the characteristic tree $\mathrm{T}$ and accompanying cycle $\mathrm{C}$ in $\mathrm{O}(\mathrm{n})$ time. The main idea of this algorithm is as following:

1. Find a planar embedding $\mathrm{H}^{\prime}$ of $\mathrm{H}$;

2. For each face $\mathrm{F}$ of $\mathrm{H}^{\prime}$, search the boundary cycle $\mathrm{C}$ of F:

If all vertices on $\mathrm{C}$ have degree 3 and deleting the edges of $\mathrm{C}$ from $\mathrm{H}$, the resulting graph is a tree $\mathrm{T}$, then $\mathrm{T}$ is the characteristic tree and $\mathrm{C}$ is the accompanying cycle.

For terminology and notation not defined in this paper, the reader is referred to [1]

\section{THE ALGORITHM}

First, we give an algorithm to determine whether a Halin graph $\mathrm{H}$ has a cubic subgraph $\mathrm{H}^{*}$ as following: 


\section{Algorithm 1:}

1. Choose an inner vertex $u$ as the root of the characteristic tree $\mathrm{T}$ of the input Halin graph $\mathrm{H}$;

2. Do the postorder traversal of $T$ rooted at $u$ as following:

3 . If the currently visited vertex $v$ is the center of a fan but not $\mathrm{u}$, then

(3.1) If $v$ has at least 4 children in the current $\mathrm{T}$, then $\mathrm{H}$ has no cubic subgraph, and the algorithm answers "No" and exits; else

(3.2) If $v$ has precisely 3 children in the current $T$, then the algorithm deletes the edge between $\mathrm{v}$ and its father in $\mathrm{T}$; else (3.3) If $v$ has precisely 2 children in the current $T$, then the algorithm keeps the edge between $\mathrm{v}$ and its father in $\mathrm{T}$; else 4. If the currently visited vertex $v$ is an inner vertex of the original $\mathrm{T}$ but not $\mathrm{u}$, then

(4.1) If $\mathrm{v}$ has at least 4 children in the current $\mathrm{T}$, then $\mathrm{H}$ has no cubic subgraph, and the algorithm answers "No" and exits; else

(4.2) If $\mathrm{v}$ has precisely 3 children in the current $\mathrm{T}$, then the algorithm deletes the edge between $\mathrm{v}$ and its father in $\mathrm{T}$; else (4.3) If $\mathrm{v}$ has precisely 2 children in the current $\mathrm{T}$, then the algorithm keeps the edge between $\mathrm{v}$ and its father in $\mathrm{T}$; else

(4.4) If $\mathrm{v}$ has precisely 1 child in the current $\mathrm{T}$, then $\mathrm{H}$ has no cubic subgraph, and the algorithm answers "No" and exits; else

(4.5) If $\mathrm{v}$ has no child in the current $\mathrm{T}$, then the algorithm deletes the edge between $\mathrm{v}$ and its father in $\mathrm{T}$ and also deletes $\mathrm{v}$ from $\mathrm{T}$; else

5. If the currently visited vertex $\mathrm{v}$ is $\mathrm{u}$, then

(5.1) If $\mathrm{v}$ has precisely 3 children or no child ( if no child, the algorithm deletes $\mathrm{v}$ from $\mathrm{T}$ ), then $\mathrm{H}$ has a cubic subgraph $\mathrm{H}^{*}$ $=\mathrm{T} \cup \mathrm{C}$, where $\mathrm{T}$ is currently obtained by the algorithm.

(5.2) Otherwise $\mathrm{H}$ has no cubic subgraph, and the algorithm answers "No" and exits.

\section{CORRECTNESS AND TIME COMPLEXITY}

Next, we prove the correctness of Algorithm 1.

Theorem 1: If a Halin graph $\mathrm{H}$ has a cubic subgraph, then Algorithm 1 succeeds to find a cubic subgraph $\mathrm{H}^{*}$ of $\mathrm{H}$; otherwise Algorithm 1 gives answer "No" .

Proof. Let $\mathrm{u}$ be the root of the characteristic tree $\mathrm{T}$ of $\mathrm{H}$ with the root $\mathrm{u}$ at the top and the tree $\mathrm{T}$ below. Let the level number of the lowest leaves in $\mathrm{T}$ be 0 , the level numbers from bottom to top in $\mathrm{T}$ be $0,1,2, \ldots, \mathrm{L}$, where $\mathrm{L}$ is the level number of $u$. If a vertex $v$ is at level 1 , then all of its children are at level $1-1$. We proceed by induction on level number 1 to prove that when Algorithm 1 visits a vertex $\mathrm{v}$ at level 1 , either the degree of $\mathrm{v}$ becomes 3 or 0 (if 0 , then $\mathrm{v}$ is deleted from $\mathrm{T}$ ) or $\mathrm{H}$ has no cubic subgraph. We prove Claim 1 first.

Claim 1: If $\mathrm{H}$ has a cubic subgraph $\mathrm{H}^{*}$, then all leaves of the original $\mathrm{T}$ are in $\mathrm{H}^{*}$

Since in $H$, every leaf of $T$ has degree 3 , if $T$ has a leaf $x$ not belonging to $\mathrm{H}^{*}$, then the leaf $\mathrm{y}$ of $\mathrm{T}$ adjacent to $\mathrm{x}$ in $\mathrm{H}$ has degree less than 3 , and hence y does not belong to $\mathrm{H}^{*}$. If $\mathrm{y}$ does not belong to $\mathrm{H}^{*}$, then the leaf $\mathrm{z}$ of $\mathrm{T}$ adjacent to $\mathrm{y}$ in $\mathrm{H}$ will have degree less than 3 , and hence $\mathrm{z}$ does not belong to $\mathrm{H}^{*}$, and so on. Then all leaves of T do not belong to $\mathrm{H}^{*}$.
But deleting all leaves from $\mathrm{T}$, only an isolated vertex of $\mathrm{T}$ remains or $\mathrm{T}$ has a vertex of degree 1 (a new leaf). The new leaf does not belong to $\mathrm{H}^{*}$ since it has degree 1 . Repeatedly deleting new leaf from $\mathrm{T}$, in the end, only one isolated vertex of $\mathrm{T}$ remains. So $\mathrm{H}$ has no cubic subgraph. By the above argument, if $\mathrm{H}$ has a cubic subgraph, then all leaves of $\mathrm{T}$ are in $\mathrm{H}^{*}$.

Now we make induction on the level number 1 of currently visited vertex $\mathrm{v}$ of $\mathrm{T}$.

When $1=0$, the vertex $\mathrm{v}$ at level 0 is a leaf of the original $\mathrm{T}$. When Algorithm 1 visits $\mathrm{v}$, it does not do anything, and $\mathrm{v}$ has degree 3 in $\mathrm{T} \cup \mathrm{C}$

Assume that when $1 \leqslant \mathrm{k}$ and Algorithm 1 visits a vertex $\mathrm{V}$ at level 1 , either the degree of $\mathrm{v}$ becomes 3 or 0 (if $0, \mathrm{v}$ is deleted from $\mathrm{T}$ ) or $\mathrm{H}$ has no cubic subgraph.

If $\mathrm{H}$ has no cubic subgraph, according to Algorithm 1, it will not visit any vertex at level $\mathrm{k}+1$ in $\mathrm{T}$. Now suppose that Algorithm 1 visits a vertex $\mathrm{v}$ at level $\mathrm{k}+1$ in $\mathrm{T}$. We have 3 cases:

Case 1: $\mathrm{v}$ is a leaf of the original $\mathrm{T}$.

Then Algorithm 1 does nothing, so $\mathrm{v}$ remains in $\mathrm{T}$ and has degree 3 in $\mathrm{T} \cup \mathrm{C}$.

Case 2: $\mathrm{v}$ is an inner vertex of the original $\mathrm{T}$ but not the root u.

By induction hypothesis, all descendants of $\mathrm{v}$ have degree 3 or 0 (if 0 , it is deleted from $\mathrm{T}$ ) by the process of Algorithm 1. Suppose that after the process of Algorithm 1, v has $p$ children $w_{1}, w_{2}, \ldots, w_{p}$.

Case (2.1): $\mathrm{p} \geqslant 4$.

But in $\mathrm{H}^{*}, \mathrm{v}$ has to be of degree 3 . So one edge between $\mathrm{v}$ and its child $w_{q}$ must be deleted. By induction hypothesis, $w_{q}$ and all its descendants (including some leaves of the original $\mathrm{T}$ ) have degree 3 in current $\mathrm{T} \cup \mathrm{C}$. Deleting the edge $v w_{q}$, the degree of $w_{q}$ becomes less than 3. So the edges between $w_{q}$ and its children must be deleted. Then the children of $w_{q}$ have degree less than 3 respectively and the edges between them and their children must be deleted. Repeatedly to do this, in the end, one leaf of the original $\mathrm{T}$ which is a descendant of $\mathrm{v}$ has degree less than 3 and must be deleted from $\mathrm{H}^{*}$. By Claim $1, \mathrm{H}$ has no cubic subgraph.

Case (2.2): $\mathrm{p}=3$.

In this case, Algorithm 1 deletes the edge between $\mathrm{v}$ and its father in $\mathrm{T}$, so $\mathrm{v}$ has degree 3 in the current $\mathrm{T}$.

Case (2.3): $\mathrm{p}=2$.

Now Algorithm 1 keeps the edge between $\mathrm{v}$ and its father, so $\mathrm{v}$ has degree 3 in the current $\mathrm{T}$.

Case (2.4) $\mathrm{p}=1$.

Including the edge between $\mathrm{v}$ and its father, $\mathrm{v}$ has degree 2 , so $\mathrm{v}$ does belong to $\mathrm{H}^{*}$, and we must delete the edge between $\mathrm{v}$ and its child $w_{q}$. Applying the argument in Case (2.1), $w_{q}$ and all its descendants (including some leaves of the original T) must be deleted from $\mathrm{H}^{*}$, by Claim $1, \mathrm{H}$ has no cubic subgraph. 
Case (2.5): $\mathrm{p}=0$.

Now Algorithm 1 deletes the edge between $\mathrm{v}$ and its father, so $\mathrm{v}$ has degree 0 and is deleted from $\mathrm{H}^{*}$.

Case 3: $v$ is the root $u$ of $T$.

Suppose that after the process of Algorithm 1, v has $p$ children in the current $\mathrm{T}$.

Case (3.1): $\mathrm{p}=3$ or 0 .

By induction hypothesis, the descendants of $\mathrm{v}$ in the original $\mathrm{T}$ have level number $1<\mathrm{k}+1$, their degrees are either 3 or 0 (if 0 , it is deleted from $\mathrm{T}$ ) and all leaves of the original $\mathrm{T}$ has degree 3 . So $\mathrm{H}$ has a cubic subgraph $\mathrm{H}^{*}=\mathrm{T}$ $\cup \mathrm{C}$, where $\mathrm{T}$ is currently obtained (by deleting $\mathrm{v}$ if $\mathrm{v}$ has 0 child).

Case (3.2): $p \neq 3$ or 0 .

Then Algorithm 1 deletes at least one edge between $\mathrm{v}$ and its child $w_{q}$. By the argument of Case (2.1), $w_{q}$ and all its descendants (including some leaves of the original $\mathrm{T}$ ) must be deleted from $\mathrm{H}^{*}$, by Claim $1, \mathrm{H}$ does not have cubic subgraph. []

Now we analyze the time complexity of Algorithm 1.

Theorem 2: In the worst case, Algorithm 1 has time complexity $\mathrm{O}(\mathrm{n})$, where $\mathrm{n}$ is the number of vertices of $\mathrm{H}$.

Proof. Algorithm 1 does postorder traversal of the characteristic tree $\mathrm{T}$ and visits each vertex once. When it visits a leaf of $T$, it does nothing. When it visits an inner vertex $\mathrm{v}$ of $\mathrm{T}$, it visits at most all vertices adjacent to $\mathrm{v}$ once, and it needs $\mathrm{O}\left(d_{T}(v)\right)$ time. For visiting the whole tree $\mathrm{T}$, it needs $\mathrm{O}\left(\sum_{v \in V(T)} d_{T}(v)\right)=\mathrm{O}(2 \mathrm{~m}(\mathrm{~T}))=\mathrm{O}(2(\mathrm{n}-1))=\mathrm{O}(\mathrm{n})$ time, where $m(T)$ is the number of edges of $T$ and $n=|V(T)|$ $=|\mathrm{V}(\mathrm{H})| . \quad[]$
The space that Algorithm 1 needs is also $\mathrm{O}(\mathrm{n})$.

\section{REFERENCES}

[1] J. A. Bondy and U. S. R. Murty, Graph Theory with Applications, Macmillan Press, London, 1976.

[2] J. A. Bondy, “Pancyclic graphs: Recent Results” , Infinite and Finite Sets, Coll. Math. Soc. János Bolyai, vol. 10, pp. 181-187, 1975.

[3] J. A. Bondy and L. Lovász, “Lengths of cycles in Halin graphs” , Journal of Graph Theory, vol. 8, pp. 397-410, 1985.

[4] G. Cornuejols, D. Naddef and W. Pulleyblank, "Halin graphs and the traveling salesman problem” , Math. Programming, vol. 16, pp. 287-294, 1983

[5] M. R. Garey and D. S. Johnson, Computers and Intractability - A Guide to the Theory of NP-Completeness, W. H. Freeman and Company, 1979.

[6] R. Halin, “ Studies on minimally n-connected graphs ” , Combinatorial Mathematics and its Applications, Academic Press, London, pp. 129-136, 1971

[7] Y. Li, D. Lou and Y. Lu, "Algorithms for the optimal Hamiltonian path in Halin graphs” , Ars Combinatoria, vol. 87, pp. 235-255, 2008 .

[8] Dingjun Lou, “Hamiltonian paths in Halin graphs” , Mathematica Applicata (Chinese), vol. 8, pp. 158-160, 1995.

[9] Dingjun Lou and Huiquan Zhu, "A note on max-leaves spanning tree problem in Halin graphs " , Australasian Journal of Combinatorics, vol. 29, pp. 95-97, 2004.

[10] Dingjun Lou and Hongke Dou, “A linear time algorithm for optimal bottleneck traveling salesman problem on a Halin graph" , Proc. 2011 International Conference on Computer, Communication and Information Technology (ICCCIT 2011), 2011, pp. 60-62.

[11] J. M. Phillips, P. Punnen and S. N. Kabadi, “A linear time algorithm for the bottleneck traveling salesman problem on a Halin graph," , Information Processing Letter, vol. 67, pp. 105-110, 1998. 